\title{
TBL en un curso de Derecho de la Universidad de Barcelona: mejora de indicadores académicos
}

\author{
TBL on a Course of Law at the University of Barcelona: Improving \\ Academic Indicators \\ Daniel Moraga \\ Facultad de Educación y Ciencias, Universidad Iberoamericana de Ciencias y Tecnología. Chile. \\ dmm2640@gmail.com
}

Antoni Font

Facultad de Derecho, Universidad de Barcelona. España. afont@ub.edu

\begin{abstract}
Resumen
El presente artículo expone la comparación de tres estrategias instruccionales aplicadas simultáneamente en un curso de grado de la Facultad de Derecho de la Universidad de Barcelona en España en el semestre otoño 2012-2013 (septiembre-enero).

Los estudiantes del curso "Derecho de la empresa y del mercado" (rectius, competencia y propiedad Industrial) fueron separados en tres grupos denominados $\mathrm{M}, \mathrm{O}$ y $\mathrm{T}$, donde el grupo $\mathrm{M}$ recibió clases magistrales (docencia tradicional), el grupo $\mathrm{O}$ recibió docencia orientada y el grupo $\mathrm{T}$ fue organizado usando la nueva estrategia de aprendizaje activo y colaborativo de Aprendizaje Basado en Equipos (TBL). Los resultados sumativos de calificaciones en las pruebas y de satisfacción de los estudiantes por el curso a través de una encuesta final de evaluación del curso, fueron registrados y comparados entre los tres grupos.

Los resultados mostraron que el grupo TBL logró mejores calificaciones que los grupos $\mathrm{O}$ y M. Siendo la diferencia entre los resultados del grupo $\mathrm{T}$ con los de ambos grupos $\mathrm{O}$ y M, altamente significativa (Chi-Square $=66.719, \mathrm{df}=4 ; \mathrm{p}=0.000)$. Consecuentemente, el análisis opuesto indica que los estudiantes de los grupos $\mathrm{O}$ y $\mathrm{T}$ tuvieron los más altos porcentajes de reprobación, $74 \%$ y $44 \%$ respectivamente, mientras que los estudiantes organizados con la estrategia TBL tuvieron un porcentaje significativamente más bajo de reprobación, de un $6 \%$. Los resultados comparativos de la encuesta final de satisfacción estudiantil en los 3 grupos de estudiantes tuvo un comportamiento similar a los resultados sumativos, con una tendencia de mayor satisfacción por el uso de la estrategia TBL versus los promedios históricos de satisfacción en las versiones previas del curso.

En conclusión, el TBL generó mejores rendimientos en este curso de Derecho, bajando significativamente las tasas de reprobación, aumentando los niveles de las calificaciones y aumentando la satisfacción estudiantil por encima de las otras 2 estrategias. Los resultados globales de este estudio piloto son claros y coincidentes con otros estudios realizados en otras áreas del conocimiento (ingenierías, salud, ciencias básicas, ciencias sociales) y permiten confirmar la superioridad de las estrategias activas y participativas como el TBL por encima de la docencia tradicional.
\end{abstract}

\section{Palabras clave}

Aprendizaje Basado en Equipos, TBL, Innovación académica.

\footnotetext{
Abstract

The present paper shows a comparison between three different instructional strategies for learning. The three strategies were simultaneously applied in a bachelor course in the
} 
autumn semester 2012 (September-January) at the Law School at Barcelona University in Spain.

The students of the course "Undertaking and Market Law" (rectius, Intellectual Property and Competition Law) were divided in three different groups, named $\mathrm{M}, \mathrm{O}$ and $\mathrm{T}$. The group $\mathrm{M}$ got simple lectures (conventional lecturing); the group $\mathrm{O}$ got an oriented learning and the group $\mathrm{T}$ was organised using the new strategy of active and collaborative learning of Team Based Learning (TBL). The student's performance in the 3 different groups was compared using final score and satisfaction survey.

The results showed that the students of the T group achieved higher scores than the students of the groups $\mathrm{O}$ and $\mathrm{M}$. The difference of the results of the groups $\mathrm{O}$ and $\mathrm{M}$ related to the results of the group $\mathrm{T}$ was strongly significant $($ Chi-Square $=66.719 ; \mathrm{df}=4 ; \mathrm{p}=0.000$. The opposite analysis is true, with fails. The percentage of fails with oriented learning or Lecturing was high, $74 \%$ and $44 \%$ respectively, while with TBL strategy, students fails drop till $6 \%$. The comparative result for satisfaction survey in the three groups also shows a tendency of higher student's satisfactions when TBL strategy is used versus historical averages of satisfaction in the previous versions of the course.

In conclusion, TBL generated better performances in this course of law, dropping significantly the rate of fails and increasing final higher scores, and consequently increases student's satisfaction with TBL as compared with the other 2 strategies. The results of this pilot-study are clear and consistent with other studies in other knowledge areas (Engineering, Health, Basic Sciences, Social Sciences). This confirms the superiority of active and participatory strategies as the TBL in front of the traditional teaching.

\section{Key words}

Team Based Learning, TBL, Innovation on teaching

\section{Introducción.}

Aun cuando la docencia del Derecho tradicionalmente ha tenido un carácter convencional de clases frontales, centradas en el profesor, existen diversos ejemplos de innovación docente que han ido enriqueciendo y aumentando las opciones didácticas en la docencia del Derecho y muy posiblemente logren instalarse como alternativas de elección en un futuro cercano. Algunas Facultades (Maastricht y York) han hecho cambios completos en su currículo, instalando estrategias activas de aprendizaje basado en problemas (ABP) en todas sus asignaturas. Otro número creciente de Facultades de Derecho en Europa, Norteamérica, Asia y Oceanía, no declaran las estrategias activas como parte de su modelo pedagógico institucional, pero han ido paulatinamente adoptando estrategias activas en uno o más cursos aislados debido a esfuerzos personales de docentes innovadores (Dana, 2007; Sparrow \& Sova McCabe, 2012). Un ejemplo destacado en España radica en el Departamento de Derecho Mercantil, Derecho del Trabajo y de la Seguridad Social de la Facultad de Derecho de la Universidad de Barcelona (Font, 2004; Font 2007), donde el profesor Font ha estado innovando en docencia y uso de TICs con aprendizaje basado en problemas (ABP) y aprendizaje basado en equipos (TBL) en su curso de "Derecho de la empresa y del mercado". Este trabajo describe los resultados de comparar tres estrategias docentes (Docencia orientada, TBL y Lecciones magistrales) en dicho curso en el semestre de otoño del curso académico 2012-2013 (septiembre-enero).

\section{Materiales y Métodos.}

\section{A. Datos del Curso.}

La Facultad de Derecho de la Universidad de Barcelona ofrece 1 vez al año el curso de

TBL en un curso de Derecho de la Universidad de Barcelona: mejora de indicadores académicos. Daniel Moraga y Antoni Font.

Página 2 de 14 
"Derecho de la empresa y del mercado" (rectius, propiedad industrial y competencia) en el semestre otoño 2012-2013 (septiembre-enero) para los estudiantes de tercer año del grado. Este curso es de carácter obligatorio para obtener el título de graduado en Derecho en un lapso de 4 años. Entre los años 2006-2007/2012-2013 este curso tuvo una matrícula cercana a 400 estudiantes, que fueron divididos en un número variable de grupos (4-9 grupos) cada uno con un rango variable de estudiantes (10-70 estudiantes).

\section{B. Organización de los Grupos del Curso.}

Este curso fue organizado en tres formatos de docencia, incluyendo Docencia Tradicional (grupo M), Docencia Orientada (grupo O), y Docencia TBL (grupo T).

i. Grupo M, clases magistrales, docencia tradicional: Los estudiantes recibieron 4 horas de clase a la semana distribuidas en dos bloques de 2 horas cada una, en días distintos y en un formato tradicional de lecciones magistrales, centradas en el profesor y en la enseñanza. Había un bajo nivel de retroalimentación a los estudiantes y sus rendimientos y aprendizajes son conocidos sólo al final de la calificación de los exámenes finales.

ii. Grupo O, docencia orientada: Los estudiantes recibieron 1 sesión intensiva de 1 hora semanal para preguntas y respuestas. No hubo propiamente clases en sentido tradicional y la retroalimentación hacia los rendimientos y aprendizajes se conocieron sólo al final de la calificación de los exámenes finales.

iii. Grupo T, estrategia TBL: Los estudiantes fueron organizados bajo la metodología de Aprendizaje Basado en Equipos, TBL. El grupo estaba compuesto de 69 estudiantes, organizados en doce equipos con un solo facilitador (Antoni Font), todos trabajando en una misma sala. Dada la naturaleza activa y participativa del TBL los estudiantes recibieron una alta retroalimentación de sus aprendizajes, se beneficiaron de la colaboración e interdependencia positiva en los equipos y junto con aprender la disciplina lograron desarrollar habilidades blandas de trabajo en equipo, tolerancia, empatía y discusión intra-equipos, entre otras.

\section{Evaluación de los grupos O, M y T.}

La evaluación de los grupos O y M se realizó un vez terminada la instrucción con exámenes finales.

La evaluación del grupo T incluyó:

- Evaluación del proceso de aseguramiento del aprendizaje inicial (PAAI). La nota PAAI $=50 \%$ prueba individual de entrada $(\mathrm{iRAT})+50 \%$ prueba grupal de entrada (gRAT).

- La evaluación de la actividad de aplicación, incluyó heteroevaluación de la resolución del problema con una rúbrica; autoevaluación con una rúbrica y coevaluación (evaluación de pares) que se calculó con la siguiente fórmula:

- Nota individual de la coevaluación $=$ Nota Grupal* $(\mathrm{w} \%+(100 \%-\mathrm{w} \%) * \mathrm{EC}$, dónde:

- Nota Grupal = Es la calificación asignada por el tutor al grupo.

- $\mathrm{w} \%=$ Es el porcentaje de la nota individual respecto de la nota grupal.

TBL en un curso de Derecho de la Universidad de Barcelona: mejora de indicadores académicos. Daniel Moraga y Antoni Font. 
- $\mathrm{EC}=\mathrm{ECTI} / \mathrm{ECTM}(\mathrm{EC}$ es un factor que proviene de las calificaciones de los pares)

- ECTI = La suma de puntos recibidos por todos los pares del equipo

- $\mathrm{ECTM}=$ El valor medio de ECTI de todos los miembros del equipo.

\section{Llenado de los grupos O, M y T.}

Los nuevos estudiantes tuvieron la posibilidad de elegir libremente el grupo en el que querían realizar sus estudios (grupos $\mathrm{O}, \mathrm{M}$ o $\mathrm{T}$ ), de acuerdo con sus preferencias pedagógicas, horarios disponibles y posibles preferencias por un profesor. Los estudiantes repetidores no tuvieron otra opción que integrarse en el grupo $\mathrm{O}$.

\section{E. Rendimiento y satisfacción.}

Para comparar los rendimientos en los tres grupos, los resultados sumativos de las calificaciones obtenidas por los estudiantes fueron agrupados en tres categorías como sigue:

- $\quad$ Puntaje de Reprobación = 0-4.9 puntos;

- $\quad$ Puntaje Suficiente = 5.0-6.9 puntos;

- $\quad$ Puntaje Superior $=7.0-10$ puntos.

Para comparar la satisfacción de los estudiantes por las tres diferentes estrategias instruccionales, se les pidió contestar a dos preguntas con una escala de $0-10$, siendo 0 el grado de menor satisfacción y 10 el de mayor satisfacción.

- La respuesta con mayor grado de satisfacción fue: "En general, estoy satisfecho con la calidad del trabajo realizado por el docente".

- La segunda respuesta con mayor grado de satisfacción fue: "La metodología desarrollada en este curso fue adecuada".

\section{F. Descripción General de la Estrategia TBL utilizada.}

La estrategia TBL (del inglés: “Team-based learning") es de naturaleza constructivista (Hrynchak \& Batty, 2012), y se fundamenta esencialmente en la teoría de aprendizaje experiencial de Kolb (Roberson \& Franchini, 2014). TBL estructura las actividades del aula en equipos, permitiendo la inversión efectiva del aula (Wallace, Walker, Braseby \& Sweet, 2014), y orientando efectivamente la docencia hacia aprendizaje activo centrado en el estudiante (Fink, 2003). El TBL ofrece a los estudiantes la oportunidad de aplicar el conocimiento conceptual a través de una secuencia de actividades que incluye trabajo individual, trabajo en equipo y retroalimentación inmediata (Parmelee, Michaelsen, Cook \& Hudes, 2012). Se puede usar en clases grandes (100 o más estudiantes) o pequeñas (menos de 25 estudiantes), con un solo profesor y dónde los estudiantes se agrupan en equipos de 5 a 7 personas en una sola sala de clases (con sillas y mesas fijas o móviles). El TBL se sustenta en 4 principios fundamentales (Michaelsen et al. 2002):

1. Equipos. Los equipos de estudiantes (de 5 a 7 integrantes) son formados por el docente, distribuyendo todos los activos del curso lo más homogéneamente posible. Los equipos tienen carácter permanente para optimizar las opciones de cohesión y evolución como equipos de aprendizaje autogestionados. 
2. Fase 1. Los equipos son preparados durante el proceso de aseguramiento del aprendizaje inicial (fase 1), para alcanzar el éxito en las actividades de aplicación (fase 2).

3. Fase 2. Los equipos de estudiantes aplican en el aula sus conocimientos en las actividades de aplicación (fase 2). Los equipos van aprendiendo, evolucionando y cohesionándose para resolver problemas reales, auténticos e interesantes, aplicando en la práctica los conceptos e ideas del curso. De esta manera, los estudiantes se ven obligados a tomar en equipo decisiones complejas, realizan reportes públicos y reciben retroalimentación inmediata y de calidad. Posteriormente, en las discusiones entre los equipos se propicia un espacio para deliberar y profundizar en los aprendizajes logrados.

4. Responsabilidad. Los estudiantes son estimulados a ser responsables de su desarrollo individual y del funcionamiento óptimo del equipo. El desarrollo individual se induce con lecturas previas y pruebas individuales al inicio de la clase, y la contribución al equipo se logra con la participación en las pruebas de los equipos, las apelaciones y las actividades de aplicación. La evaluación entre pares (coevaluación) resulta ser un elemento esencial para motivar la responsabilidad y participación de los estudiantes en los equipos.

Aun cuando el TBL está altamente centrado en el estudiante y en el aprendizaje activo, en comparación con el ABP posee un mayor grado de estructuración y control por parte del profesor respecto de los temas a discutir. El sistema de actividades y de evaluación del TBL favorece la alta participación y compromiso de todos los estudiantes, generando un apropiado reconocimiento diferencial de la participación y contribución de los estudiantes al trabajo del equipo (Michaelsen, Knight \& Fink, 2002; Michaelsen, Sweet \& Parmelee, 2009). Cada curso TBL tiene entre cinco y siete módulos de TBL. La secuencia típica de un módulo de TBL incluye un proceso de aseguramiento del aprendizaje inicial, seguido de tres a cinco clases con actividades de aplicación.

\section{G. Proceso de Aseguramiento del Aprendizaje Inicial.}

1. Lectura pre-clase. Las lecturas incluyen típicamente 30-50 páginas de libros, papers, reportes o monografías, vídeos, etc. Puede ser valioso entregar una guía de la lectura, si los estudiantes no están familiarizados con los contenidos a tratar. Para las lecturas es válido el concepto "menos es más".

2. Prueba individual de aseguramiento del aprendizaje inicial (iRAT). Al inicio de la clase, los estudiantes responden individualmente esta prueba iRAT, la cual consiste generalmente de 7 a 20 preguntas de opción múltiple o de respuesta corta u otros formatos evaluativos. La existencia de iRAT obliga a una lectura anterior a la clase e induce la adquisición inicial de conocimientos fundamentales del curso a nivel individual.

3. Prueba grupal de aseguramiento del aprendizaje inicial (gRAT). Una vez terminada la iRAT, los alumnos deben resolver la misma prueba, pero ahora en equipos, de ahí su denominación, gRAT. Con ello el proceso de aseguramiento del aprendizaje se fortalece gracias a los aportes

TBL en un curso de Derecho de la Universidad de Barcelona: mejora de indicadores académicos. Daniel Moraga y Antoni Font. 
individuales y a la discusión grupal y al hecho de poder resolver las dudas originadas en la iRAT y encontrar las respuestas correctas de forma colectiva. Esto básicamente "legaliza la copia" y establece un escenario ideal de aprendizaje colaborativo. Otro aspecto clave de la gRAT, cuando la prueba es de opción múltiple, consiste en el uso de la técnica de retroalimentación inmediata (IF-AT) con tarjetas de "raspe y gane". Los equipos deben negociar y argumentar cuál de las alternativas van a raspar. $\mathrm{Si}$ aparece una $\mathrm{x}$, los estudiantes saben que han logrado la respuesta correcta (¡hay mucha alegría en el grupo!); si no es así, deben discutir otras variables y elegir otra alternativa. Con ello, se completa todo el ciclo de un proceso de aprendizaje (lectura, iRAT y gRAT) y cada alumno termina conociendo de forma inmediata y con discusión profunda la respuesta correcta a cada una de las preguntas.

4. Apelación grupal de las respuestas incorrectas. Al término de las pruebas iRAT/gRAT, es posible que los equipos hayan encontrado errores de contenido o de constructo en las pruebas, y dada la naturaleza activa del aprendizaje, el instructor estimula a los equipos a apelar las preguntas que fueron respondidas de forma incorrecta y que ellos consideran que contenían algún error. Esto orienta a los equipos a discutir, encontrar y fundamentar en su material de lectura o en otras fuentes de información (por ejemplo, una página web) la racionalidad de las apelaciones. Este proceso de apelación permite defender sus argumentos y profundizar en sus aprendizajes.

5. Mini-clase. Al concluir el proceso de aseguramiento del aprendizaje inicial, el instructor realiza una mini-clase en torno a los conceptos más complejos o que fueron más problemáticos para los estudiantes al responder a los iRAT/gRAT. Con ello se cierra la fase de aseguramiento del aprendizaje inicial y los estudiantes están ya preparados para aplicar sus nuevos conocimientos en las actividades de aplicación.

Después del Proceso de Aseguramiento del Aprendizaje Inicial, la mayor parte del tiempo del módulo en aula, se usa por los equipos de estudiantes para las Actividades de Aplicación (Roberson \& Franchini, 2014), en las que se aplican los conceptos del curso y se resuelven problemas; todo ello orientado a lograr los resultados de aprendizaje del módulo.

\section{H. Actividades de Aplicación en módulo TBL.}

Las actividades de aplicación se caracterizan por incluir: a) un problema significativo y relevante de la disciplina; b) el trabajo simultáneo de todos los equipos en el mismo problema; c) la elección específica de una o más alternativas que resuelven el problema y que han sido predeterminadas como opciones plausibles por el docente; y d) el reporte simultáneo: los equipos reportan en forma simultánea y visible la elección específica que han tomado.

1. Problema significativo. Los equipos trabajan en un problema significativo y relevante, donde la calidad del problema condiciona en forma decisiva la efectividad de la actividad de aplicación. Los problemas deben obligar al uso de conceptos del curso y estar relacionados con los resultados de aprendizaje. Para ello, se puede utilizar un diseño retroactivo: primero,

TBL en un curso de Derecho de la Universidad de Barcelona: mejora de indicadores académicos. Daniel Moraga y Antoni Font. 
centrarse en el resultado de aprendizaje que se quiere lograr; a continuación, elegir una lectura orientada a este resultado de aprendizaje; seguidamente, el contenido de la prueba iRAT debe estar alineado con estos conceptos; y finalmente, se debe generar uno o varios problemas complejos pero factibles de ser resueltos con los conocimientos alcanzados.

2. Mismo problema. Los equipos trabajan el mismo problema. Al entregar el mismo problema a todos los equipos se genera una atmósfera muy proactiva, que crea oportunidades de reflexión, defensa y discusión de ideas, y formas de enfrentar y resolver un problema. Se facilita la comparabilidad y la discusión entre los grupos. Cuando se realiza trabajo grupal con problemas distintos y reporte secuencial, se genera una atmósfera de baja energía, dónde los estudiantes no están necesariamente motivados para examinar el proceso de pensamiento y decisiones presentadas por los otros grupos.

3. Elección específica. Los equipos deben tomar decisiones específicas orientadas a resolver el problema. El uso de preguntas abiertas ha sido tradicionalmente el sustento de grandes esfuerzos docentes para estimular el pensamiento crítico en los estudiantes. No obstante, el uso de preguntas abiertas y complejas podría ser un desafío demasiado grande para algunos profesores y es un proceso muy emergente que toma mucho tiempo. El aspecto más cuestionable del uso de preguntas abiertas es su ineficiencia en el momento de dejar que los alumnos reporten sus respuestas y la dificultad para comparar sus respuestas con las de los pares. En contraposición, la elección específica del TBL genera la oportunidad de guiar a los estudiantes a comprometerse con una posible solución y estar preparados para defender/argumentar dicha decisión, siendo esto una de las mayores fortalezas de las actividades de aplicación del TBL.

4. Reporte simultáneo. Los equipos reportan en forma simultánea sus decisiones. Un reporte simultáneo se logra simplemente levantando públicamente una carta que indica una elección específica de cada equipo. Cuando un equipo particular, observa que otro equipo ha tomado una decisión diferente, naturalmente ocurre una comparación de racionalidad y de toma de decisiones. Se genera debate entre los equipos, incluyendo la defensa de su propia postura y el desafío hacia la decisión del otro equipo. Este reporte requiere que los equipos articulen sus pensamientos con los otros equipos, colocando sus pensamientos en palabras. Esto ayuda a la construcción de aprendizajes profundos y significativos. La retroalimentación de los pares es inmediata y está enfocada a responder preguntas del tipo “¿cómo llegaste a esa decisión?” antes que “¿cuál es la respuesta correcta?”.

\section{Resultados.}

La comparación de los rendimientos sumativos en los tres grupos del estudio, $\mathrm{O}$, M y T del curso "Derecho de la empresa y del mercado" en la Facultad de Derecho de la Universidad de Barcelona se ofrece en la figura 1. Los resultados mostraron que casi la mitad $(46 \%)$ de los estudiantes organizados en docencia TBL (grupo T) lograron puntajes superiores, mientras que sólo porcentajes muy bajos de estudiantes que recibieron docencia orientada (grupo $\mathrm{O}, 3 \%$ ) o tradicional (grupo $\mathrm{M}, 6 \%$ ), lograron puntajes superiores, siendo la diferencia entre los resultados del grupo $\mathrm{T}$ y los de ambos grupos $\mathrm{O}$ y $\mathrm{M}$ altamente significativa (Chi-Square=66.719; $\mathrm{df}=4 ; \mathrm{p}=0.000$ ). El

TBL en un curso de Derecho de la Universidad de Barcelona: mejora de indicadores académicos. Daniel Moraga y Antoni Font. 
coeficiente de contingencia generado por estos datos $(\mathrm{C}=0.538 ; \mathrm{n}=164 ; \mathrm{p}=0.000)$ puede ser interpretado como una relación moderada entre ambas variables.

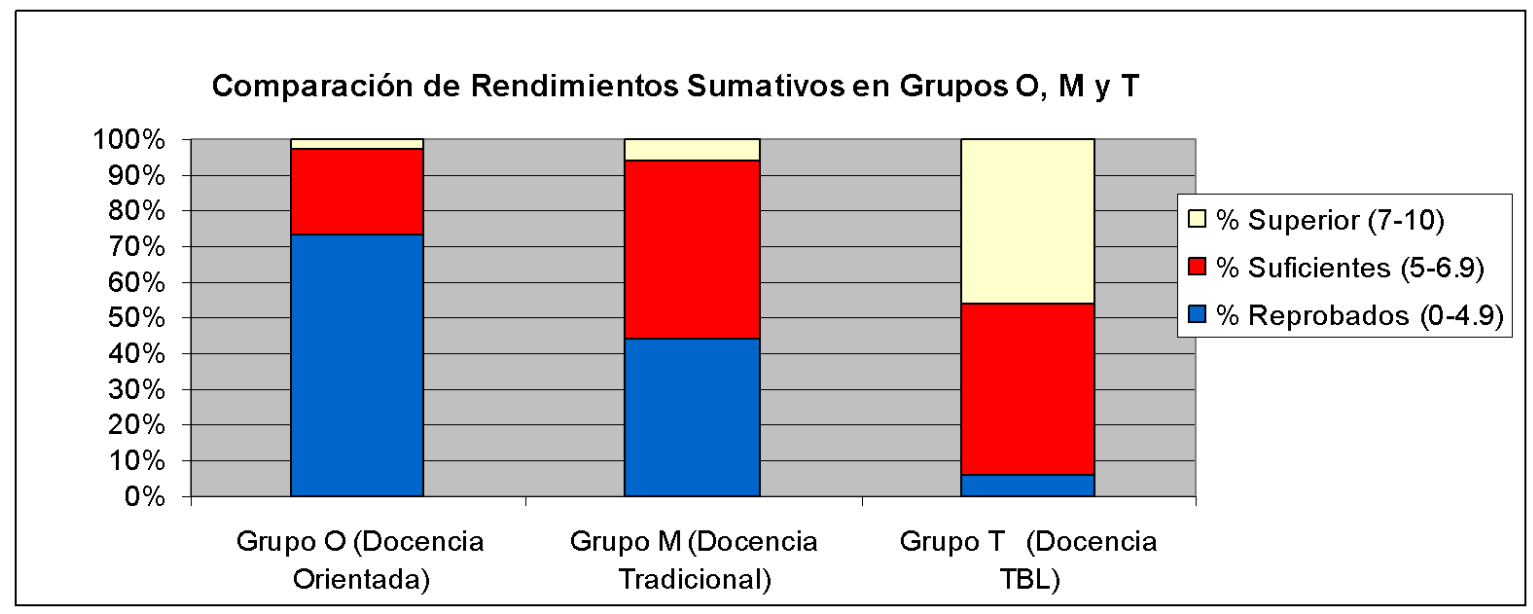

Figura $\mathbf{n}^{\circ}$ 1: Comparación de los rendimientos sumativos en los 3 grupos M, O y T (Tradicional, Orientado y TBL) de acuerdo a los resultados sumativos. El porcentaje relativo de estudiantes que están en una de las tres categorías de las calificaciones obtenidas se representa en colores como sigue: Color Amarillo, porcentaje con puntaje superior = 7.0-10 puntos; Color Rojo, porcentaje con puntaje suficiente (5.0-6.9 puntos; y Color Azul, Porcentaje con puntaje de reprobación (0-4.9 puntos).

Consecuentemente, el análisis opuesto indica que los estudiantes de los grupos Orientado (grupo O) y Tradicional (grupo M) obtuvieron los porcentajes más altos de reprobación, $74 \%$ y $44 \%$ respectivamente, mientras que los estudiantes organizados con la estrategia TBL (grupo T) obtuvieron un porcentaje de reprobación del $6 \%$, significativamente más bajo.

El análisis histórico (2006-2007/2011-2012) de los rendimientos sumativos de los estudiantes del mismo curso, se muestra en la figura $\mathrm{n}^{\circ} 2$. El análisis comparativo de los rendimientos sumativos históricos con los resultados del grupo TBL periodo 2012-2013, reveló que la estrategia TBL genera resultados siempre mejores que los históricos para este curso. La relación entre las variables es estadísticamente significativa (ChiSquare $=12.458, \mathrm{df}=4 ; \mathrm{p}=0.014)$. 


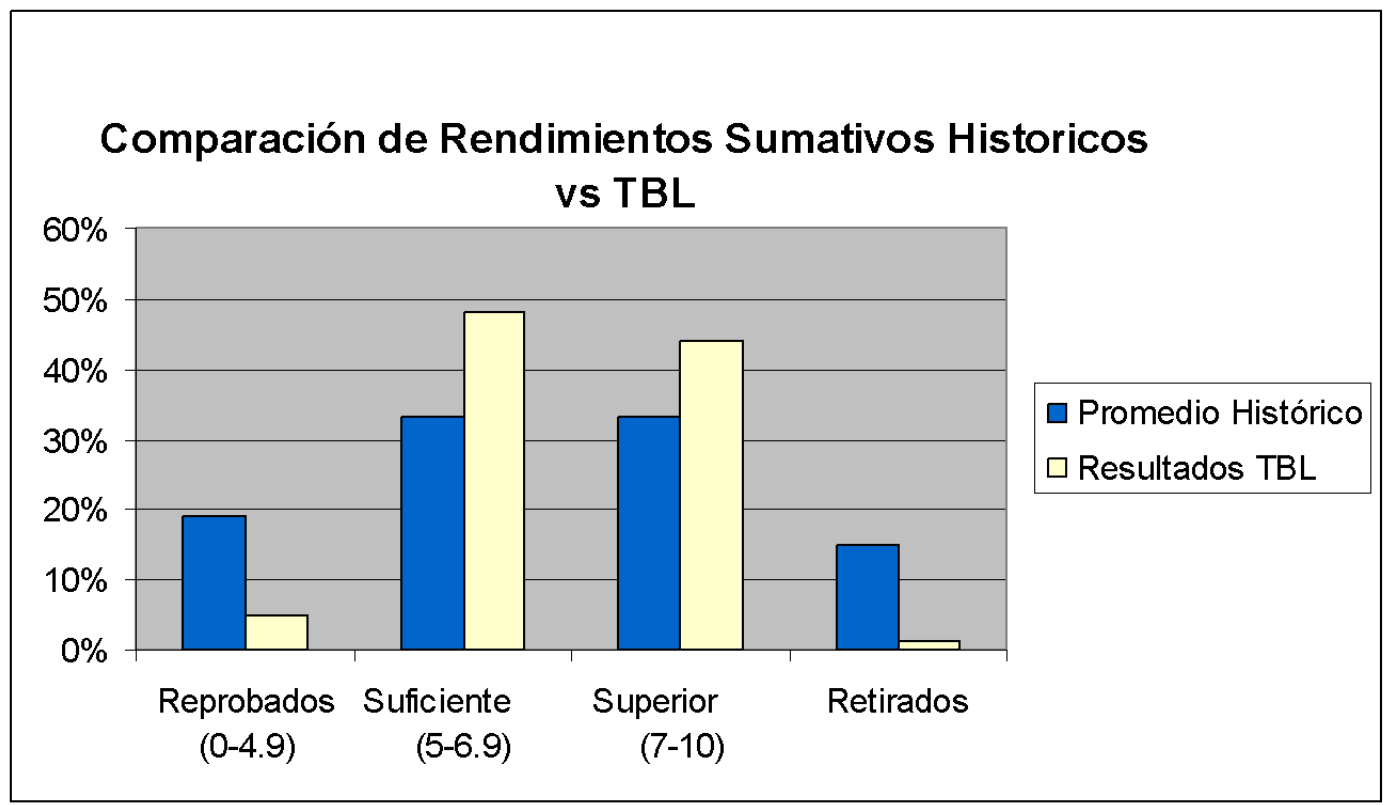

Figura $\mathbf{N}^{\circ}$ 2: Comparación de los rendimientos promedios históricos del curso "Derecho de la empresa y del mercado" en el periodo (2006-2007/2011-2012) en color azul, versus los resultados TBL 2012-2013 en color amarillo. La relación entre las variables es estadísticamente significativa (Chi-Square=12.458, $\mathrm{df}=4 ; \mathrm{p}=0.014)$, aún cuando el coeficiente de contingencia $(\mathrm{C}=0.071 ; \mathrm{n}=2,446 ; \mathrm{p}=0.014)$ debe ser interpretado como una baja relación entre las variables.

Los resultados comparativos de la encuesta final de satisfacción estudiantil en los 3 grupos de estudiantes del curso 2012-2013, se ofrecen en la Figura $n^{\circ}$ 3. De forma similar a los resultados sumativos, el TBL muestra una clara tendencia de mayor satisfacción por el uso de esta estrategia frente a los promedios históricos de satisfacción estudiantil de los años 2006-2007 a 2011-2012, tanto respecto a la calidad del trabajo realizado por el docente, (color azul) como respecto de si la metodología desarrollada en el curso fue adecuada (color amarillo).

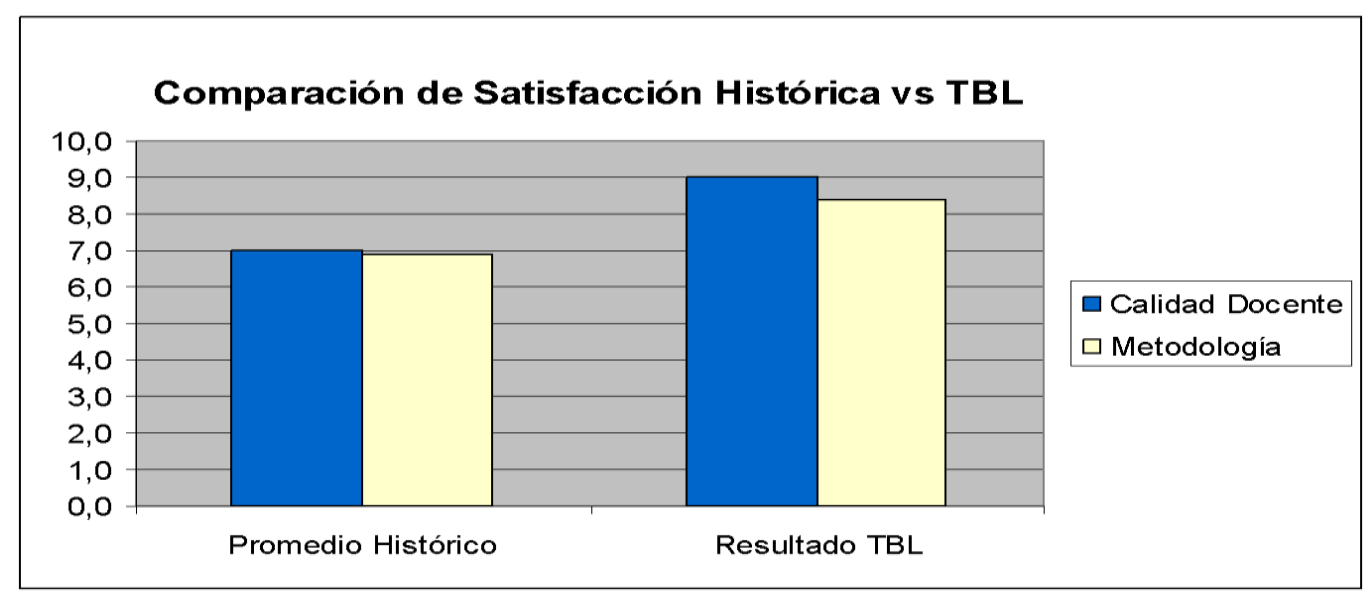

Figura $\mathrm{n}^{\circ}$ 3. Comparación de los resultados históricos en la encuesta de satisfacción del curso "Derecho de la empresa y del mercado" en el periodo (2006-2007 / 2011-2012), versus los resultados de satisfacción TBL 2012-2013. Se muestra una clara tendencia de mayor satisfacción por el uso de la estrategia TBL versus los promedios históricos de satisfacción estudiantil 2006-2007 / -2011-2012, tanto respecto a la calidad del trabajo realizado por el docente, (color azul) como respecto de si la metodología desarrollada en el curso fue adecuada (color amarillo).

TBL en un curso de Derecho de la Universidad de Barcelona: mejora de indicadores académicos. Daniel Moraga y Antoni Font.

Página 9 de 14 


\section{Discusión}

El presente artículo expone la comparación de resultados sumativos de tres estrategias instruccionales (docencia tradicional, docencia orientada y docencia TBL) aplicadas simultáneamente en el curso de "Derecho de la empresa y del mercado" en la Universidad de Barcelona durante el periodo 2012-2013. Los resultados muestran que TBL generó mejores rendimientos en este curso de Derecho, bajando significativamente las tasas de reprobación, aumentando los niveles de las calificaciones y aumentando la satisfacción estudiantil por encima de las otras dos estrategias de docencia orientada y tradicional. La alta eficiencia y efectividad y los bajos costos mostrados por el TBL en este curso de Derecho, impulsó la adopción del TBL en otros cursos del Departamento de Derecho Mercantil, Derecho del Trabajo y de la Seguridad Social de la Facultad de Derecho de la Universidad de Barcelona.

El uso del TBL en la enseñanza del Derecho se ha reportado desde hace algunos años, a partir de los trabajos en USA (Dana, 2007; Sparrow, S. \& Sova McCabe, 2012). En ambos casos se describe la racionalidad del uso e implementación del TBL en las aulas de Derecho y los desafíos administrativos que ello impone, pero no agregan datos sumativos comparativos, con las otras estrategias de aprendizaje tradicional y vigente en las facultades y escuelas de Derecho en el mundo, como en este trabajo.

Algunas facultades y escuelas de Derecho como Maastricht y York han hecho cambios completos en su currículo, declarando e instalando estrategias activas de aprendizaje basado en problemas (ABP) en todas sus asignaturas. Esto ha impuesto una alta carga financiera en estas instituciones, para poder implementar grupos pequeños de $\mathrm{ABP}$ con un solo tutor, en una sola sala y con capacitación ad hoc de sus plantas docentes en ABP. En otro número creciente de Escuelas y Facultades de Derecho en Europa, Norteamérica, Asia y Oceanía, no existe una declaración institucional de estrategias activas, como parte de su modelo pedagógico, pero debido a los esfuerzos personales de docentes innovadores como Antoni Font, la docencia en dichas instituciones ha ido paulatinamente adoptando estrategias activas en uno o más cursos aislados.

A pesar de los excelentes resultados logrados con el uso de la estrategia ABP en diversas instituciones de nivel mundial en Europa, Norteamérica, Asia y Oceanía, el uso del ABP no ha sido tan amplio en Latinoamérica y África y en general en instituciones menos solventes, principalmente debido a los altos costes económicos asociados con esta estrategia de aprendizaje. Por esta razón, parece razonable estimular el uso de estrategias activas y colaborativas más abordables desde el punto de vista financiero pero con excelentes resultados de eficiencia y efectividad. El TBL mantiene las mismas virtudes del trabajo colaborativo en grupos pequeños de estudiantes que poseen otras metodologías como el ABP, pero agrega algunas ventajas atractivas como la no exclusión de docencia tradicional y su menor costo por permitir razones muy elevadas estudiante/facilitador, entre otras ventajas (Michaelsen, Knight \& Fink, 2002; Michaelsen, Sweet, Parmelee, 2009, Moraga \& Font, 2013).

Es evidente que el factor económico ha restringido fuertemente el avance de innovaciones como el ABP en las facultades y escuelas de Derecho de todo el mundo. Es difícil poder llegar a modelos institucionales con uso masivo de ABP como en las facultades de

Maastricht (http://www.maastrichtuniversity.nl/web/Faculties/FL/education_law.htm) y de York (http://www.york.ac.uk/law/undergraduate/law-at-york/pbl/), por el alto costo 
económico de su implementación. No obstante, ejemplos como los aquí expuestos, que implicaron una migración desde ABP a TBL en la Facultad de Derecho de la Universidad de Barcelona, demuestran que es posible innovar en la docencia del Derecho, a bajos costos, con calidad, eficiencia y efectividad.

Estos resultados aquí mostrados para el área de Derecho son coincidentes con numerosos estudios publicados del impacto del uso de la estrategia TBL en otras áreas del conocimiento como las ingenierías, la salud, las ciencias básicas y las ciencias sociales (Haidet et al. 2014; Sweet \& Michaelsen, 2012; Nanes, 2014; Paterson \& Sneddon, 2011; Bobbitt, Inks, Kemp \& Mayo, 2000; Changa, Chenb, Wangb, Wanga \& Wuc, 2015) y permiten confirmar la superioridad de las estrategias activas y participativas como el TBL por encima de la docencia tradicional, tal como lo indica un reciente publicación de un meta-análisis de 225 estudios publicados que demuestra un incremento del $6 \%$ y $50 \%$ para calificaciones y tasas de aprobación en cursos de aprendizaje activo frente a las tradicionales clases magistrales (Freeman, et al., 2014).

Es claro que no basta con eliminar el factor económico para lograr el aseguramiento de la innovación docente. Aún hay que lidiar con la resistencia natural de los docentes al cambio. Un elemento inmanente en la resistencia al cambio en los docentes tradicionales es la llamada "pérdida de cobertura de contenidos disciplinares fundamentales", que no serían "vistos" si se cambia el foco de la docencia hacia modelos centrados en el estudiante. No obstante las evidencias existentes con el TBL refutarían esta resistencia al cambio, ya que el TBL sí favorece el uso, la práctica y el aprendizaje profundo de los contenidos disciplinares fundamentales, y agrega además competencias esenciales para un jurista. De acuerdo con Sparrow (2012): "aun cuando el TBL condiciona el uso de la mayor parte del tiempo para las actividades de aplicación en equipos, los estudiantes logran estudiar y aplicar más conceptos jurídicos que cuando un profesor usa docencia tradicional con una combinación de diálogo socrático de casos, clases cortas y tareas fuera del aula que impliquen aprendizaje activo. Al finalizar un curso de TBL, todos los estudiantes habrán trabajado reiteradamente en lo que suele hacer un jurista en la práctica, trabajar juntos para resolver un problema significativo. Al hacer esto, los estudiantes aprenden como interactuar profesionalmente con otros, construyen con los otros aprendizajes disciplinares relevantes, y aprenden de y con los demás habilidades de comunicación, resolución creativa de problemas, cómo estudiar mejor, gestionar el tiempo, y resolver conflictos".

Una de las razones fundamentales del éxito relativo del TBL como estrategia docente es su alineamiento con principios constructivistas del aprendizaje (Hrynchak \& Batty, 2012) y su bajo costo de implementación dado que no requiere alta capacitación docente, permiten tener altas razones estudiante/docente y no exigen ampliaciones o modificaciones de infraestructura para su aplicación. Junto a lo anterior, se ha estimado que el TBL casa muy bien con los 5 primeros principios de instrucción definidos por Merril (2000 y 2012) ya que logra promover el aprendizaje al 1) Involucrar a los estudiantes en la resolución de problemas del mundo real; 2) generar activación del conocimiento previo para sustentar y fundamentar el nuevo conocimiento; 3) ofrecer actividades de aplicación en el aula con problemas relevantes y significativos con varias opciones de solución, una de las cuales es la más correcta; 4) obligar a los estudiantes a tomar decisiones tanto en la fase de aseguramiento del aprendizaje inicial como en las actividades de aplicación para aplicar sus conocimientos; y 5) familiarizar y aterrizar las

TBL en un curso de Derecho de la Universidad de Barcelona: mejora de indicadores académicos. Daniel Moraga y Antoni Font.

Página 11 de 14 
problemáticas disciplinares en las actividades de aplicación para lograr la integración con el mundo del estudiante.

En el siglo pasado, la estrategia de aprendizaje activo que lideró las innovaciones educativas en todo el mundo y que incluso logró penetrar en la docencia del Derecho, fue el aprendizaje basado en problemas (ABP). Pero el cambio metodológico desde el ABP al TBL ha comenzado a ocurrir en otras instituciones además de lo observado en la Facultad de Derecho de la Universidad de Barcelona. En la actualidad el mayor lugar de implementación del TBL en el mundo está en las escuelas y facultades de Medicina, siendo un ejemplo paradigmático el del mejor rendimiento de los estudiantes de la escuela de Medicina de la Universidad de DUKE, en Singapur, que usa la estrategia TBL en comparación con el de los estudiantes de la escuela de Medicina de la misma Universidad de DUKE, en USA, con docencia tradicional (Kamei, et al., 2012). Una muestra de la relevancia e intromisión del TBL en el mundo, es la creciente cantidad de libros, artículos, videos y páginas web relacionadas con la difusión del TBL, que se plasma muy bien en la siguiente frase: "es una locura el interés por el TBL en las escuelas de Medicina de Europa" (Larry Michaelsen, comunicación personal, septiembre 2014). El TBL está concitando gran interés en las Escuelas de Medicina del mundo y en todas las áreas del saber. Situación equivalente a lo ocurrido con el ABP en los pasados 40 años. Pero ahora, hay interés por migrar desde ABP a TBL o al menos combinar lo mejor de ambas estrategias (Dolmans, et al, 2014).

Los resultados globales de este estudio piloto son claros y coincidentes con otros estudios realizados en otras áreas del conocimiento (ingenierías, salud, ciencias básicas, ciencias social Belli, S. y Reyes) en http://www.um.es/ead/red/47es) y permiten confirmar la superioridad de las estrategias activas y participativas como el TBL por encima de la docencia tradicional (Freeman et al. 2014).

Presentación del artículo: 19 de enero de 2016 Fecha de aprobación: 7 de marzo de 2016 Fecha de publicación: 30 de abril de 2016

Moraga, D. y Font, A. (2016). TBL en un curso de Derecho de la Universidad de Barcelona: mejora de indicadores académicos. RED. Revista de Educación a Distancia. 49(3). Consultado el (dd/mm/aaaa) en http://www.um.es/ead/red/49

\section{Referencias}

Bobbitt, M., Inks, S., Kemp, K. \& Mayo, D. (2000). "Integrating Marketing Courses to Enhance Team-Based Experiential Learning". Journal of Marketing Education, vol.22, 15 .

Changa, C.; Chenb, Y., Wangb, J., Wanga, S. \& Wuc, C. (2015). "The case study of team-based learning methodology with teachers of four domains in the senior high school". Procedia - Social and Behavioral Sciences, vol.176, 804 - 810. Recuperado de http://www.sciencedirect.com/science/article/pii/S1877042815005807, consultado en junio 2015. 
Dana, S. (2007). "Implementing Team-Based Learning in an Introduction to Law Course”. Journal of Legal Studies Education, vol.24, n.1, 59-108.

Dolmans, D., Michaelsen, L., van Merrienboer, J. \& van der Vleuten, C. (2015). "Should we choose between problem-based Learning and team-based Learning? No, combine the best of both worlds!" Medical Teacher, 37 (4), 354.

Fink, D. (2003). Creating Significant Learning Experiences: An Integrated Approach to Designing College Courses. San Francisco: Jossey-Bass.

Font, A. (2004). "Líneas maestras del aprendizaje por problemas". Revista Interuniversitaria de Formación del Profesorado, 18 (1), 81-97.

Font, A. (2007). "El uso de las TIC como soporte para el ABP”. En: La metodología del Aprendizaje Basado en Problemas. http://www.ub.edu/dikasteia/LIBRO_MURCIA.pdf

Freeman, S., Eddy, S., McDonough, M., Smith, M., Okoroafor, N., Jordt, H. \& Wenderoth, M.(2014). "Active learning increases student performance in science, engineering, and mathematics". PNAS (Proceedings of the National Academy of Sciences), vol. 111 (23) 8410-8415.

Haidet, P., Kubitz, K. \& McCormack, W. (2014). "Analysis of the team-based learning literature: TBL comes of age". Journal on Excellence in College Teaching, vol.25, n.3\&4, 303-333. http://celt.muohio.edu/ject/issue.php?v=25\&n=3\%20and\%204, (consulta, junio 2015).

Hrynchak, P \& Batty, H (2012). "The educational theory basis of team-based learning". Medical Teacher. 2012; 34(10):796-801. doi: 10.3109/0142159X.2012.687120. Epub 2012 May 30.

Kamei, R., Cook, C., Puthucheary, J., \& Starmer, S. (2012). "21st Century Learning in Medicine: Traditional Teaching versus Team-based Learning”. Medical Science Educator, Volume 22:No. 2 - articles.

Michaelsen, L., Knight, B., \& Fink, D. (2002). Team-Based Learning: A Transformative Use of Small Groups for Large and Small Classes. Westport, Conn: Bergin \&Garvey.

Michaelsen, L., Sweet, M. \& Parmalee, D. (2009). "Essential Elements of Team-Based Learning”. Team-Based Learning: Small Group Learning's Next Big Step. New Directions in Teaching and Learning, 7-27. Traducción al español como "Elementos Esenciales del Aprendizaje Basado en Equipos” de D. Moraga, O. López y J.G. Díaz, en:

http://www.teambasedlearning.org/Resources/Documents/AprendizajeBasadoenEqui pos.pdf

Merril, D. (2000). First Principles of Instruction. Obtenido desde: https://www.indiana.edu/ tedfrick/aect2002/firstprinciplesbymerrill.pdf

TBL en un curso de Derecho de la Universidad de Barcelona: mejora de indicadores académicos. Daniel Moraga y Antoni Font. 
Merril, D. (2012). First principles of instruction. John Wiley and Sons.

Moraga, D. \& Font, A. (2013), "Comparación entre tres principales formas de instrucción educativa". Actas del quinto congreso nacional de docencia en ciencias jurídicas. Valencia, 11-13 de septiembre, 2013, 289. Consultable en: http://www.uv.es/innodret/pub/2013actas_congreso.pdf.

Nanes, K. (2014). "A modified approach to team-based learning in linear algebra courses". International Journal of Mathematical Education in Science and Technology, vol.45, n.8, 1208-1219.

Parmelee D., Michaelsen, L., Cook, S. \& Hudes, P. (2012). Team-based learning: A practical guide: AMEE Guide No. 65. Medical Teacher; 34: e275-e287.

Paterson, J. \& Sneddon J. (2011). "Conversations about curriculum change: Mathematical thinking and team-based learning in a discrete mathematics course". International Journal of Mathematical Education in Science and Technology, vol.42, n. 7, 879-889.

Roberson, B. \& Franchini, B. (2014). "Effective task design for the TBL classroom". Journal on Excellence in College Teaching, vol.25, n.3\&4, 275-302. Recuperado de http://celt.muohio.edu/ject/issue.php? $\mathrm{v}=25 \& \mathrm{n}=3 \% 20$ and $\% 204$, consultado en junio 2015.

Sparrow, S. \& Sova McCabe, M. (2012). “Team-Based Learning in Law”. Journal of Legal Writing Institute, vol.53, 153-208.

Wallace, M., Walker, J., Braseby, A. \& Sweet, M. (2014). "Now, what happens during class? Using team-based learning to optimize the role of expertise within the flipped classroom". Journal on Excellence in College Teaching, vol.25, n.3\&4, 253-273. http://celt.muohio.edu/ject/issue.php? $\mathrm{v}=25 \& \mathrm{n}=3 \% 20$ and\%204 (consulta: junio 2015). 